\title{
Nanomaterials: A Foundational Building Block to Nanotechnology Breakthroughs Past and Future
}

\author{
Daniel S. Gianola and Kevin J. Hemker
}

Opinion: For Your Consideration

Always interested in hearing the thoughts of our readers, JOM offers For Your Consideration as a forum for individual opinions and thoughtful discourse on topics pertaining to JOM coverage areas. Find out more about this occasional feature at http://www.tms.org/pubs/journals/JOM/authorTypesofPapers.aspx. Opinions expressed are the author's and not necessarily those of TMS or the editorial staff.

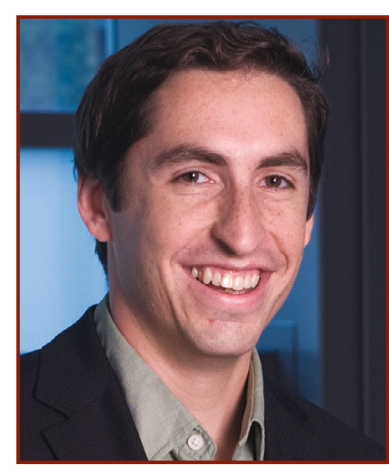

Daniel S. Gianola

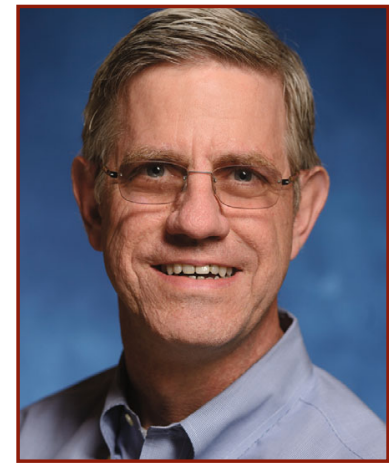

Kevin J. Hemker
The National Nanotechnology Initiative (NNI) was launched in 2001 to promote a broad interagency goal of understanding and controlling materials at the nanoscale to produce disruptive advances in technology with tangible societal benefits. NNI's 15th anniversary, to be observed in October 2016, provides a salient opportunity to reflect on past successes and future prospects.

It is our view that over the course of NNI's service, the early hype of nanotechnology has been supplanted by tangible applications ranging from medical theragnostics, to high-performance computers and data storage, to high efficiency energy conversion and storage devices. In the early days of NNI, news articles on nanotechnology outpaced the number of awarded patents, but that trend has been reversed and successful nanotechnology patents and products now outnumber popular news stories. ${ }^{1,2}$ For a more concrete example, consider the story of Novak Djokovic, who this year became the first male tennis player in recent memory to hold all four major championships simultaneously. His equipment of choice? A racquet frame enhanced by graphene, the 2D allotrope of carbon celebrated for its high strength and stiffness. The field of nanoscience is still in its adolescence and, while scientific questions about the fundamental nature of materials at the nanoscale persist, nanotechnology is poised to have a massive societal impact.

Take, for instance, the nanotechnologyinspired grand challenges listed by the U.S.
National Academy for Sciences, a response to the call-for-arms from the White House Office of Science and Technology Policy:

(1) Increase the five-year survival rates by 50 percent for the most difficult to treat cancers; (2) Create devices no bigger than a grain of rice that can sense, compute, and communicate without wires or maintenance for 10 years, enabling an "internet of things" revolution; (3) Create computer chips that are 100 times faster yet consume less power; (4) Manufacture atomically precise materials with 50 times the strength of aluminum at half the weight and the same cost; (5) Reduce the cost of turning sea water into drinkable water by a factor of four; and (6) Determine the environmental, health, and safety characteristics of a nanomaterial in a month. ${ }^{3}$

These challenges represent complex multidisciplinary issues, with nanostructured materials underpinning many of the proposed solutions. In a similar vein, consider the European Commission's two major flagship programs, Graphene and the Human Brain Project. One is explicitly a nanomaterial, while the other places nanomaterials in the mix as solutions to scalable computing and novel conformable sensors for understanding neurological networks and pathways.

We should also reflect on the great progress that has been made to date. The focus on nanoscience has greatly advanced our ability to synthesize, characterize, and model nanomaterials with unprecedented 


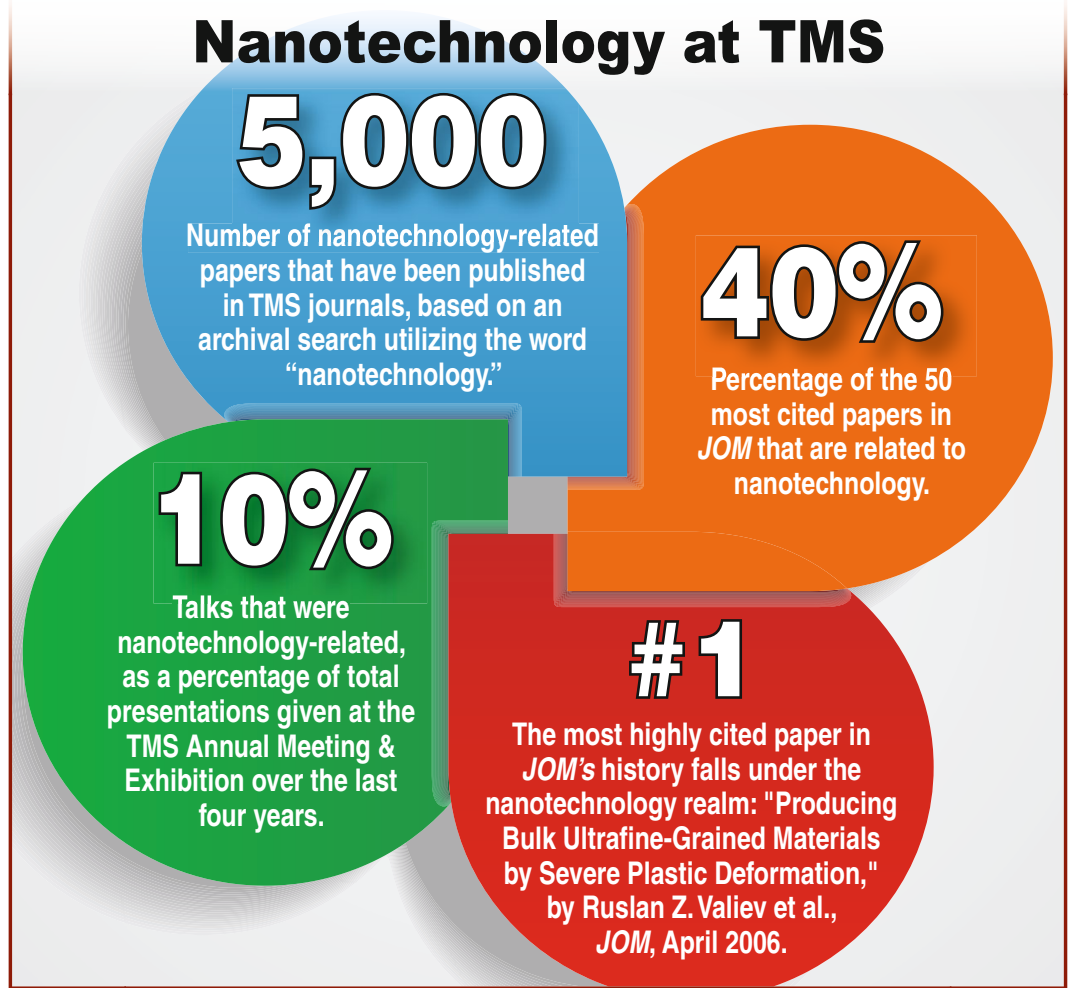

Figure 1: A snapshot of TMS's contributions supporting the advancement of nanomaterials. physical and chemical properties that are derived from dimensional constraints. In applications where engineered components must bear load, structural nanomaterials show clear promise and take advantage of "smaller is stronger" trends. Owing to a scarcity of defects, many nanomaterials can withstand elastic stresses near their theoretical limit. Examples of integration of nanoscale building blocks into meso- and macroscale materialsthe enrichment of polymer matrix composites with carbon nanotubes, the development of nanocrystalline ceramics, and the incorporation of nanoprecipitate strengthened alloys, to name a few possibilities - hold great promise and are expected to deliver unprecedented properties. However, challenges in bridging nano- to mesoscale fabrication, characterization, and modeling remain.

Functional materials - such as electrodes for rechargeable batteries, active light harvesting materials for photon to electrical energy conversion, and thermal management materials - have all made use of nanostructuring to match physical length scales associated with transport of electrons, ions, and magnetic spins. Consider thermoelectrics, which convert a temperature gradient to an electrical current and where a resurgence in interest is predicated on scavenging of waste heat. Nanoscaled materials - judiciously sized to fall between the electron and phonon mean free paths for facile transport of the former and abundant scattering of the latter-show large materials figures of merit relative to their bulk counterparts. In analogy to structural materials, the highest performing thermoelectrics rely on precipitating nanoscale phases coherent with an active matrix that at once scatter heat-carrying phonons and energetically align with flowing charge carriers.

TMS and its members have invested in and played a significant role to advance the maturation of nanoscience and the development of nanomaterials. Figure 1 illustrates the activity and impact that TMS and its members are having, but our work is not done.

Sustained scientific and technology efforts are required that make use of the collaborative environment fostered by international materials societies such as TMS and its partners. Investments in nanotechnology have paid undeniable dividends, and the stage is set for continued investments to yield spectacular results.

Daniel S. Gianola is currently associate professor in the Materials Department at the University of California, Santa Barbara, where his research group specializes in the microand nanomechanical behavior of materials. He has been a TMS member since he was a graduate student, and has been involved in co-organizing several symposia at TMS annual meetings and Materials Science and Technology conferences. He received the TMS Early Career Faculty Fellow Award in 2013.

Kevin J. Hemker is the Alonzo G. Decker Chair and Professor of Mechanical Engineering at Johns Hopkins University, holding joint appointments in the Departments of Materials Science and Engineering and Earth \& Planetary Sciences. Currently the Vice President of the TMS Board of Directors, Hemker is a 2014 TMS Fellow and served two terms on the TMS Board of Directors as Public \& Governmental Affairs (P\&GA) Director from 2008 to 2013.

\section{References}

1. "Beyond the Nanohype," The Economist (2003).

2. "A Little Risky Business," The Economist (2007).

3. L. Whitman, T. Kalil, "A Call for Nanotechnology-Inspired Grand Challenges," https://www.whitehouse.gov/ blog/2015/06/17/call-nanotechnologyinspired-grand-challenges.

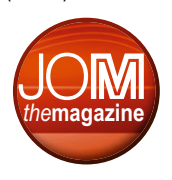

